\title{
Chimeric recombinant rotavirus-like particles as a vehicle for the display of heterologous epitopes
}

\author{
Andrea Peralta*1, Paula Molinari ${ }^{1}$ and Oscar Taboga ${ }^{1,2}$
}

\author{
Address: ${ }^{1}$ Consejo Nacional de Investigaciones Científicas y Técnicas (CONICET). Av. Rivadavia 1917 (c.p.1033) Ciudad Autónoma de Buenos \\ Aires, Argentina and 2Instituto de Biotecnología, Instituto Nacional de Tecnología Agropecuaria (INTA), cc25 (c.p.1712) Castelar, Buenos Aires, \\ Argentina \\ Email: Andrea Peralta* - avperalta@cnia.inta.gov.ar; Paula Molinari - pmolinari@cnia.inta.gov.ar; Oscar Taboga - otaboga@cnia.inta.gov.ar \\ * Corresponding author
}

Published: 6 November 2009

Virology Journal 2009, 6:192 doi:10.1186/1743-422X-6-192

This article is available from: http://www.virologyj.com/content/6/I/192

(C) 2009 Peralta et al; licensee BioMed Central Ltd.

This is an Open Access article distributed under the terms of the Creative Commons Attribution License (http://creativecommons.org/licenses/by/2.0), which permits unrestricted use, distribution, and reproduction in any medium, provided the original work is properly cited.
Received: 13 June 2009

Accepted: 6 November 2009

\begin{abstract}
In order to improve the presentation and immunogenicity of single epitopes, virus-like particles (VLPs) are being used as platforms for the display of foreing epitopes on their surface. The rotavirus major capsid protein VP6 has the ability to self-assemble into empty non-infectious VLPs. In the present study, we analyzed the use of double layered VLPs (made up of VP2 and VP6 rotavirus proteins) as carriers to display a 14 amino acid epitope fused to three different aminoacidic regions of VP6 exposed on the surface of VLPs. Although all chimeric protein were correctly expressed in insect cells, only one of them resulted in spontaneous assembly of VLPs displaying the heterologous epitope on their surface, confirmed by sandwich ELISA and electron microscopy. Furthermore, the injection of chimeric VLPs into mice elicited higher antibody titers than the monomeric chimeric protein. Our results identify an specific amino acid region of VP6 which allows the insertion of at least a 14 amino acid heterolgous epitope and demonstrate its potential as immunogenic carrier.
\end{abstract}

\section{Background}

Virus-like particles (VLPs) are complexes composed of viral structural proteins that retain the ability to selfassemble without requiring the presence of the viral genome, mimicking the overall structure of virus particles. They are considered as safe and non-infectious tools for several purposes such as diagnostic assays [1-4], cell interaction studies [5,6] and vaccines [7-14]. VLPs are commonly more immunogenic than subunit or recombinant immunogens based on single, monomeric proteins, and are able to stimulate both the humoral and cellular pathways of the immune system. VLPs offer a promising approach to the production of vaccines against many diseases, because their repetitive, high density display of epitopes is often effective in eliciting strong immune responses [15]. This is further enhanced by the particulate nature of VLPs, especially in the size range of around 40 $\mathrm{nm}$ that appears to be optimal for uptake of nanoparticles by dendritic cells [16].

VLPs provide the spatial structure for the display of conformational epitopes and can be exploited as platforms for the presentation of foreign epitopes or targeting molecules on chimeric VLPs. This can be achieved via transcriptional fusion of heterologous sequences and viral proteins in such a way that the chimeric protein is assembled into VLP during de novo synthesis.

Rotaviruses, members of the Reoviridae family of segmented, dsRNA genome, are the most important cause of viral gastroenteritis in infants and young animals around the world [17-19]. Members of this family are nonenvel- 
oped, with complex capsids containing several concentric protein layers displaying icosahedral symmetry. Rotaviruses have a triple concentric capsid. The innermost layer, which is composed of VP2 protein, encloses the different genomic segments of dsRNA together with VP1 and VP3 proteins. The middle layer is composed of 780 molecules of VP6 protein, which are distributed as 260 trimers. The outermost layer is composed of glycoprotein VP7 and spikes of dimers of VP4 [17]. Both outer proteins are the targets for neutralizing antibodies and define the virus $G$ and P serotypes, respectively. VP6 is the most abundant protein in the virus particle, comprising about of $51 \%$ of the total protein mass [17].

Co-expression of VP2 and VP6 in both mammalian and insect cells results in the production of VLPs that can be easily purified $[20,21]$, whereas the expression of VP2 alone results in the production of pseudo-core particles or CLPs $[22,23]$. Although the formation of VLPs requires the presence of VP2, VP6 alone can form spherical or tubular aggergates [24] and could be overexpressed and purified in large quantities. VP6 self-assembles into different types of particles depending on conditions such as $\mathrm{pH}$, ionic strength and divalent cation concentration.

Although VP6 is part of the middle layer of the rotavirus mature particles, it elicits a strong humoral immune responses after rotavirus infection. At least one strong Th cell epitope has been mapped, which is highly conserved in most group A rotavirus strains studied so far and it was proposed that Th cells specific for VP6 epitopes may constitute an important proportion of the total polyclonal Th cell response against a porcine rotavirus in spleen cells [25].

Based on the study of VP6 priming immune responses to the external rotavirus proteins, Esquivel et al suggested that the VP6-specific Th cells can provide cognate help to B cells specific for neutralizing epitopes on the VP7 and/ or VP4 molecules, and that this help could be heterotypic [26]. On the other hand, synthetic peptides spanning different regions of VP6 protein were able to elicit high titers of antibodies $[27,28]$.

Together, the ability of VP6 to form multimeric structures and the strong immune responses that VP6 can elicit in different species point at VP6 in an excellent candidate as a carrier for foreign epitopes. In this report, we used the baculovirus expression system to produce recombinant VLPs based on rotavirus capsid VP6 and VP2 proteins from SA1 1 strain of simian rotavirus. We first searched for positions in the surface loops of VP6 that could accommodate a foreign 14 amino-acid peptide derived from the simian paramyxovirus 5 (V5 epitope, [29]) without affecting capsid formation and then we examined whether the heterologous sequences inserted into these sites were displayed on the surfaces of the recombinant VLPs.

\section{Results}

\section{Election of possible insertion sites into VP6 sequence}

SA11 VP6 protein is $97 \%$ identity to RF VP6, In turn, RF VP6 structural conformation is well known (PDB code: 1qhd). In order to select possible insertion sites into VP6 sequence, we analyzed the spatial conformation of 1qhd and three regions located in loops exposed onto VLPs surface were selected: the amino acids 171-172 into loop A'A" and amino acids 311-312 and 301 to 308 both in loop HI. Selected regions are shown in Figure 1.

\section{Effect of epitope insertion on recombinant expression and antigenic properties of monomers}

In order to evaluate the effect of epitope insertion on the expression, interaction and antigenicity of the recombinant VP6 proteins, insertion mutants were constructed bearing 14 amino-acids of the V5 epitope at different positions and expressed in the baculovirus system.

Extracts of Sf9 cells infected with the different recombinant baculoviruses exhibited an intense band in Coomassie-stained polyacrylamide gels, at the electrophoretic mobility expected for the recombinant proteins and absent in extracts of mock-infected Sf9 cells (data not shown). The apparent molecular weights of the VP6 variants were slightly higher than wild-typeVP6 and reached similar expression levels.

To confirm the identity of the chimeric proteins, a monoclonal antibody to V5 epitope and an anti-VP6 rabbit polyclonal serum were employed in Western blot experiments. The extracts of Sf9 cells infected with all the recombinant baculoviruses exhibited a band with the expected electrophoretic mobility that strongly reacted with the monoclonal antibody and was absent in the extracts of Sf9 AcVP6-infected (Figure 2A), suggesting that the antigenicity of the V5 epitope was manteined in all the mutants. The same intense bands were observed when the polyclonal rabbit serum directed to rotavirus was used (Figure 2B), but it was possible to observe a partial proteolysis in mutants $\mathrm{VP}_{301} / \mathrm{V} 5$ and $\mathrm{VP}_{311} / \mathrm{V} 5$ which was only partially prevented with the addition of aprotonine and leupeptinA.

As $\mathrm{VP}_{311} / \mathrm{V} 5$ showed a pronounced cleavage, we continued this work only with $\mathrm{VP}_{171} / \mathrm{V} 5$ and $\mathrm{VP}_{301} / \mathrm{V} 5$.

\section{Effect of epitope insertion on trimer and multimer formation}

In order to evaluate the implicances of the insertion of V5 sequence on the ability of VP6 mutants to oligomerize into trimers, total extracts from Sf9 cells infected with the 


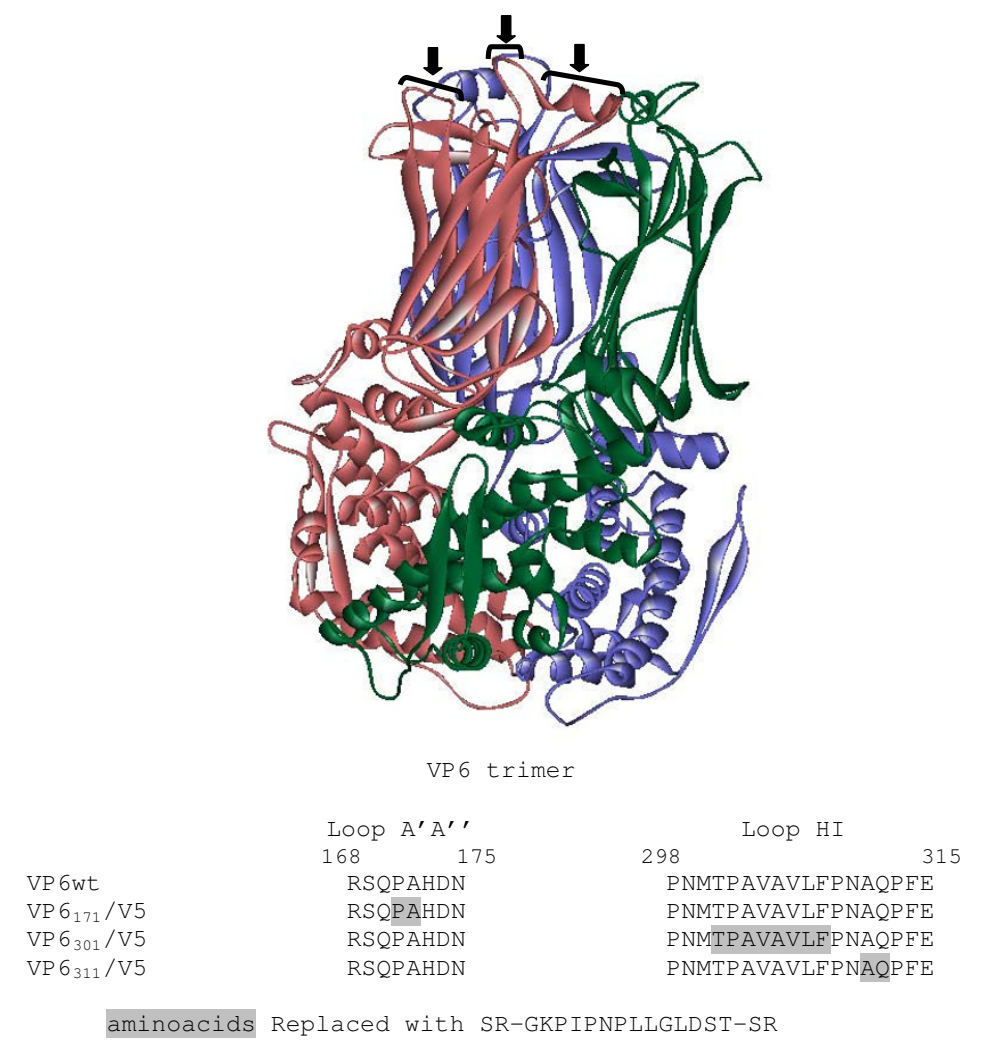

Figure I

Insertion of the epitope V5 into VP6 sequence. A solid ribbon drawing the VP6 trimer is shown at the top. Arrows and brackets show only in one chain the amino acids regions of VP6 protein selected in order to insert a heterologous epitope. In the lower panel, partial amino acid sequences of VP6 and a series of VP6 mutants were created by replacing the amino acids residues indicated by grey boxes with the V5 epitope flanked by SR residues.

different recombinant baculoviruses were disrupted at $37^{\circ} \mathrm{C}$ instead of $100^{\circ} \mathrm{C}$, resolved by gel electrophoresis (in no-reducing or native conditions) and monomers and trimers detected by Western blot using a SA11 polyclonal rabbit serum or a V5 monoclonal antibody. As control of trimers, VP6wt and mutant VP6-V5N were used. This mutant bears the epitope V5 in the amino terminal of VP6 as it has been reported that insertions at N-terminal of VP6 did not affect the ability to oligomerize into trimers (Reddy et al 1992). As previously reported [30], wild type VP6 was only detected as trimers in these conditions (Figure $2 \mathrm{C}$ ). In the same way, mutant VP6-V5N was detected as trimers. However, we could not detect trimers from insertional mutants $\mathrm{VP}_{171} / \mathrm{V} 5$ or $\mathrm{VP6}_{301} / \mathrm{V} 5$.

The ability of the insertional mutants to form multimeric structures was first evaluated by their ability to sediment through $30 \%$ sucrose cushions. Lysates of Sf9 infected cells from the different recombinant baculoviruses AcVP6/V5 were ultracentrifugated and the pellets were disrupted and resolved by SDS-PAGE and Western blot. The results showed that mutants $\mathrm{VPG}_{171} / \mathrm{V} 5$ and $\mathrm{VPG}_{301} /$ $\mathrm{V} 5$ were able to interact in some multimeric form.

\section{Effects of epitope insertion on VLP assembly and exposition of the heterologous sequence}

In order to determine the ability of the different VP6 mutants to interact with VP2 to form VLPs, supernatans of co-infections were analyzed by $\mathrm{CsCl}$ gradients and electron microcopy. By electron microscopy observation, only $\mathrm{VP6}_{171} / \mathrm{V} 5$ was able to form VLPs. These VP6 ${ }_{171} / \mathrm{V} 5$ VLPs were indistinguishable from wild type VLPs, and the amount of VLPs per field was very similar (Figure 3). $\mathrm{VP6}_{171} / \mathrm{V} 5$ VLPs also exhibited mean diameter comparable with those of VLPs VP6 (Table 1).

In order to determine the epitope exposition on VLPs $\mathrm{VP6}_{171} / \mathrm{V} 5$ surface, a sandwich ELISA was performed. The results suggested that V5 was exposed on the surface of these VLPs (Figure 4A) 
A

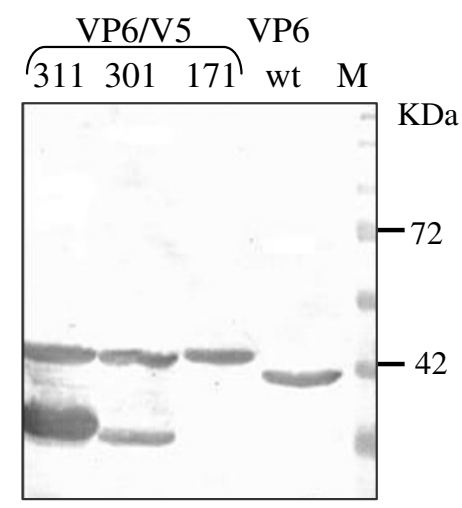

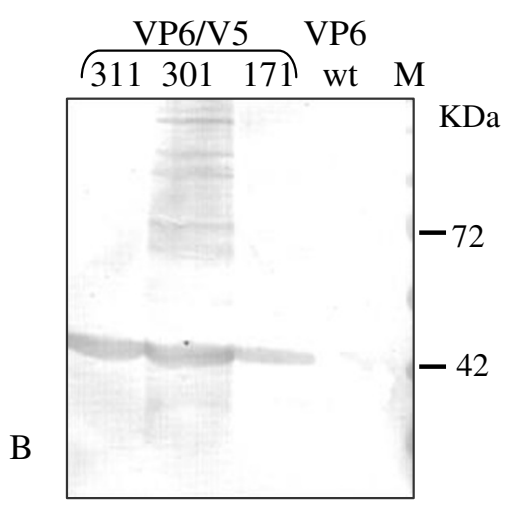

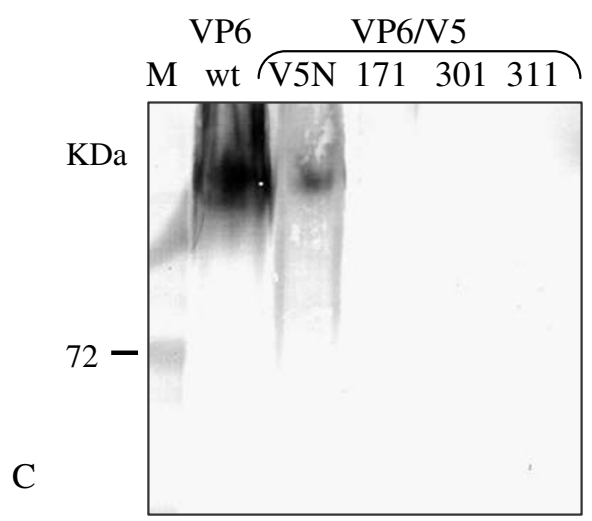

\section{Figure 2}

Expression of chimeric VP6/V5 protein. Sf9 cells infected with baculovirus $A c V P 6, A_{c V P 6}{ }_{171} / \mathrm{V} 5, \mathrm{AcVP}_{301} / \mathrm{V} 5$ or $\mathrm{AcVP6}_{31 \mathrm{I}} / \mathrm{V} 5$, were analyzed by western blot using a polyclonal sera anti-VP6 (A) or a MAb anti-V5 epitope (B). In order to determine the trimerization ability of the chimeric VP6/V5, cells extracts were analyzed by electrophoresis under native conditions and western blot $(C)$.

To further confirm these results, purified $\mathrm{VP}_{171} / \mathrm{V} 5$ VLPs were immunolabeled with MAb anti-V5 and revealed with an anti-mouse IgG conjugated to gold particles. Figure 4B shows that $\mathrm{VP6}_{171} / \mathrm{V} 5$ VLPs were specificly labeled on their surface.

Immunogenic features of the VLPs VP6 ${ }_{|7|} / V 5$ mutant As high quantities of $\mathrm{VP}_{171} / \mathrm{V} 5$ were routinely obtained rendering VLPs with V5 epitope exposed to the surface, we

Table I: Mean diameter of VLPs

\begin{tabular}{cc}
\hline VLPs $(6+2)$ & MEAN DIAMETER \\
\hline VP6wt & $63 \pm 2,6 \mathrm{~nm}$ \\
\hline$V^{2} 6_{171} / \mathrm{V} 5$ & $69 \pm 3,4 \mathrm{~nm}$
\end{tabular}

These values were obtained through analysis of 100 particles by electron microscopy. decided to continue the experiments of immunization only with this mutant. The immunogenicity of $\mathrm{VP}_{171} / \mathrm{V} 5$ VLPs was compared with the same chimeric protein as a monomer by innoculating groups of five mice with each preparation. VLPs of wild type VP6 were used to immunize control mice. Antibody titers elicited to anti V5 epitope following two immunizations are given in Table 2. High levels of anti-V5 $\left(\sim 3 \log _{10}\right)$ antibodies were measured after 20 days after booster when as little as $300 \mathrm{ng}$ of chimeric VLPs were administered, representing 9 ng of V5 peptide. In contrast, it was necessary to innoculate $5 \mu \mathrm{g}$ of chimaeric monomers to elicit detectable antibody titers to V5, suggesting that the multimeric presentation of V5 on the surface of the VLP strongly enhanced their immunogenicity.

\section{Discussion}

We demonstrated the influence of the insertion of a 14 amino acids heterologous sequence in the ability of VP6 

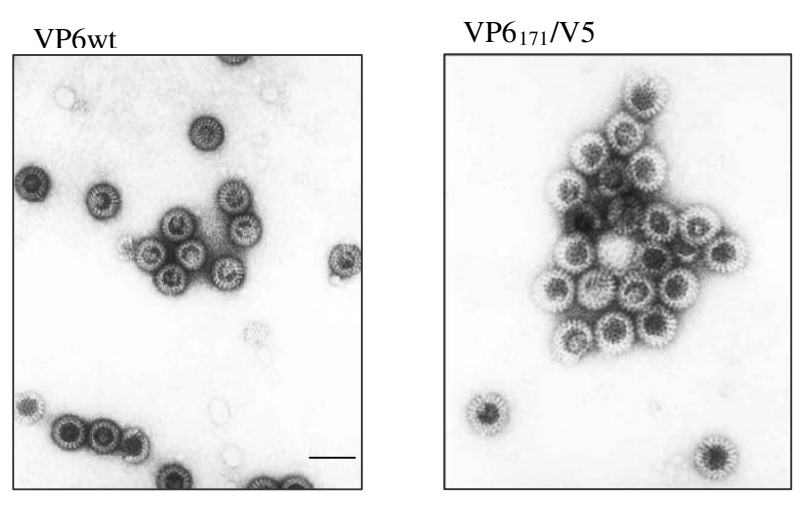

\section{Figure 3}

Negative staining of wt or recombinant VLPs. In order to characterize the VLPs formation, supernants of Sf9 cells co-infected with recombinant baculoviruses expressing VP2, VP6wt or VP6 171 / 55 or $\mathrm{VP6}_{301} / \mathrm{V} 5$ were processed as described in Materials and Methods. Particles were adsorbed onto carbon-coated grids, staining with $2 \%$ uranyl acetate and examined immediately. Bar indicates $100 \mathrm{~nm}$.

from a simian rotavirus strain to trimerize, multimerize and interact with VP2 to render chimaeric VLPs. In particular, when the V5 sequence was inserted at position 171 of VP6, VLPs were indistinguishable of recombinant 2/6 particles, with the inserted epitope protruding from the particle surface. In addition, these chimeric VLPs elicited higher antibody titers to the inserted epitope than the monomeric chimeric protein, suggesting that these chimerical VLPs may be a useful vaccine approach for the multimeric presentation of immunogenic epitopes.

The morphogenesis of rotavirus in mammalian cells has been widely studied (López et al., 2005), although some aspects remain less understood. Rotavirus VP6 protein plays a central rol in virus morphogenesis by establishing interactions with other structural proteins as well as to the nonstructural protein NSP4. Core particles assembled from VP1, VP2 and VP3 acquire an icosahedral layer of VP6 arranged in trimeric units. Double-shelled particles are then translocated to the lumen of the endoplasmic reticulum via NSP4 and virus assembly is completed by the incorporation of VP4 and VP7. The obtention of rotavirus-like particles by infection or co-infections of insect cells by recombinant baculovirus has provided strong evidences on the site of assembly of the major rotavirus structural proteins VP2 and VP6 and in the characterization of functional domains. VP2 forms pseudocores of $50 \mathrm{~nm}$ of diameter composed by 60 dimers in the cytoplasm of infected insect cells observed as aggregates [31], while VP6 alone forms tubular structures composed by a variable number of trimers [24]. However, when both recombinant proteins are simultaneously expressed, VP6 drasti- cally changes its localization [31] and self-assembly, rendering double-layered VLPs that are assembled inside the insect cell and can be recovered from the supernatants of co-infected cultures.

By the in vitro transcription and translation analysis of truncated forms of VP6, a large domain involved in trimerization have been previously characterized spanning amino-acidic positions 105 to 328 [32]. By using VP6 deletion mutants produced in mammalian cells by recombinant vaccinia virus, the trimerization domain could be delimited to a region between amino-acids 246 and 314 [30], and the $\mathrm{N}$ terminal end of this domain was postulated between amino-acids 147 and 246. Subsequently, by using X-ray crystallography approaches [33] it was postulated that the amino acids 171 and 172 of VP6 are involved in intra-trimer contacts. In this work, we observed that the insertion of the V5 sequence in mutant $\mathrm{VP}_{171}$ affects trimer formation. This result supports the hypothesis about the extension of the N-terminal end of the trimerization domain, extending it at least to the amino-acid 171 .

The analysis of the three-dimensional structure of VP6 from rotavirus group A using X-ray crystallography [33] determined that the region proposed as a trimerization domain is localized within a bigger domain formed for $\beta$ strands, denominated domain $\mathrm{H}$ (residues 151 to 334). This amino-acidic organization is characterized by its rigidity, and any insertion affecting the $\beta$-strand structure may also affect trimer formation. However, bioinformatic analysis of VP6/V5 chimeras (Swiss prot and Modeller, results not show) has predicted that the V5 insertions at positions 171-172, 311-312 and a replacement at positions 301-308 do not drastically affect the structure of domain H of the VP6 monomer. However, it was possible to obtain recombinant VLPs VP6-V5 only when the heterolgous epitope was inserted into 171-172 residues. Possibly, the insertion of an heterologous sequence in this particular amino acidic region of VP6, unstabilizes or prevents the formation of trimers, but the presence of VP2 protein counteracts this negative effect and helps in the formation of stable VLPs. This fact agrees with the results obtained by X-ray crystallography where the correct geometry of the viral particle is determined by VP2 [24,33,34].

Rotavirus-derived VLPs were first assessed as an immunological carrier by Redmond and collegues in 1991. They demonstrated that peptides coupled to VP6 spheres elicited a greater humoral response than other traditional carriers or peptides alone, and that this carrier was not supressed by the pre-existence of anti-rotavirus antibodies $[27,28]$. We demonstrated the influence of the insertion of an heterologous sequence in the ability of simian rotavirus VP6 to trimerize, multimerize and interact with VP2 


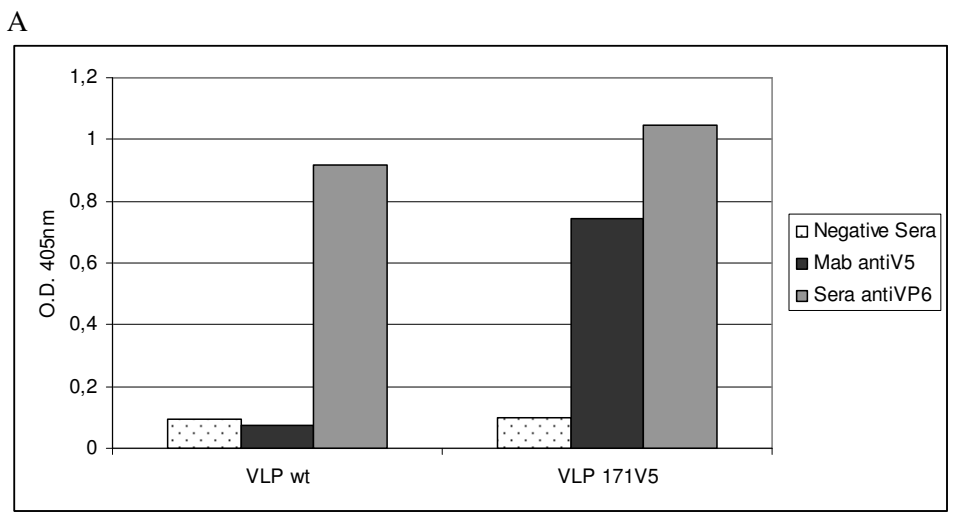

B

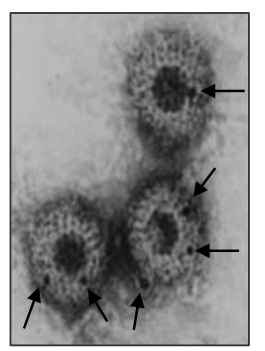

\section{Figure 4}

Characterization of recombinant VLPs VP6 ${ }_{171}$ /V5. A) Sandwich ELISA to detect epitope exposition onto recombinant VLPs surface. Purified wt and recombinant VLPs were captured by polyclonal sera against SAII strain, then were detected by a monoclonal antibody anti-V5 peptide or a polyclonal mouse sera against VP6 protein. B) Microscopy electronic and immunogold labeled. Purified recombinant VLPs VP6 ${ }_{171} / \mathrm{V} 5$ were loaded onto nickel grids were incubated with a MAb anti-V5 and then labeled with a rabbit anti-mouse conjugated with gold.

to render chimeric VLPs. In particular, when the V5 sequence was inserted at position 171-172 of VP6, VLPs were indistinguishable of recombinant $2 / 6$ particles, with the inserted epitope exposed on the particle surface. In addition, these chimeric VLPs elicited higher antibody titers to the inserted epitope than the monomeric chimaeric protein, suggesting that this chimeric VLP may be useful vaccine vehicles for the multimeric presentation of immunogenic epitopes.

Table 2: Anti-V5 epitope antibody titers elicited in mice immunized with VLPs $\mathrm{VP6}_{171} / \mathrm{V} 5$ or monomeric $\mathrm{VP6}_{171} / \mathrm{V} 5$, measured by ELISA

\begin{tabular}{lc}
\hline Immunogen & Mean $\log _{10}$ ELISA endpoint titer \\
\hline VLPs & $3.08 \pm 0.28(2.6-3.2) \dagger$ \\
Monomers & $1.88 \pm 0.40(1.7-2.6)$ \\
\hline
\end{tabular}

Titers were expressed as $\log _{10}$ of the reciprocal of the highest serum dilution, which give OD readings al least twofold higher than those of control animals. $†$ Ranges of mean titers are shown in brackets.

\section{Methods}

Primer design for insertional mutagenesis

A collection of mutants harboring an XbaI site at the desired VP6 (SA11 strain) positions were created by PCR using adequate primers and named according to the position of the insertion in the amino-acid sequence. The internal positions in the amino-acid sequence of VP6 SA11 strain were selected on the basis of the analyses of spatial conformation of VP6 RF strain deposited on Protein Data Bank (PDB code: 1qhd) and visualized using the WebLab Viewer software (Accelrys Software Inc.). Three regions located in loops exposed onto rotavirus capsid surface were selected. These amino acidic regions were 171-172, 311-312 and 301 to 308. The primers used for mutagenesis were: L171-for: TTTCTAGATTGTGATCTATTTAGTGT, L171-rev: GGTCTAGACATGATAATTTGATGGGC, L311-for: ATCTAGACCATTCGAACATCAT, L311rev: ATCTAGAATTCGGGAATAGTAC, R308-for: CCTCTAGACCGAATGCACAGCCATTC and R301-rev: TTTCTAGACATGTTTGGTGGTCTCAG. The XbaI restriction sites are in bold. 


\section{Construction of recombinant baculoviruses}

The complete methodology for the construction of recombinant baculoviruses has been previously published as a manual [35]. The VP6 and VP2 sequences used in this work derived from the simian strain SA11. For VP2 expression, VP2 coding sequence was first subcloned into pVL1393 (Pharmingen). For VP6 mutants, PCR amplification products were obtained using pBSVP6 (bearing the entire coding sequence of VP6, kindly provided by Dr. J. Blackhall) as a template. N-terminal and C-terminal moieties were sequentially cloned into pVL1393, creating an $\mathrm{Xbal}$ site at the junction and replacing two residues in VP6. For all mutants, the primer VP6-for (AAAGCTTAACATGGATGTCCTATA, bearing a HindIII restriction site) was used as the forward primer for the $\mathrm{N}$-terminal moiety and VP6 rev (TGGTACCTCATTTAATGAGCAT, bearing a KpnI restriction site) was used as the reverse primer for the C-terminal moiety. Once the two moieties of VP6 with $\mathrm{XbaI}$ sites were cloned together, a synthetic dsDNA constructed by annealing of oligonucleotides V5-For and V5Rev (V5-for: AATCTAGAGGTAAGCCTATCCСTCTCCTC, V5-rev: AATCTAGACGTAGAATCGAGACCGAGGAGAGGGTT, the XbaI restriction sites are in bold) and coding for an epitope derived from simian paramyxovirus 5 [29] was introduced in frame. An extra recombinant VP6 (called VP6-V5N) was constructed bearing the V5 epitope at the N-terminal end (The primers used were: VP6N-for: AACCCGGGGATGTCCTATACTCTTTG, VP6N-rev: AATCTAGATCATTTAATGAGCATGC, V5N-for: TCCCGGGAGTATGGCTAAGCCTATCCCTAACCCTCTCCTC, V5N-rev: TCCCGGGCGTAGAATCGAGACCGAGGAGAGGGTT. The XmaI restriction site sare in bold, the ATG codon is pointed in italic). Relevant sequences of the transfer plasmids were confirmed by sequencing and Sf9 insect cells were co-transfected with $2 \mu \mathrm{g}$ of each plasmid and $0.5 \mu \mathrm{g}$ of linear AcNPV DNA (Pharmingen) using cellfectin (Invitrogen). Resulting recombinant baculoviruses AcVP2, AcVP6, AcVP6 ${ }_{171} / \mathrm{V} 5$ AcVP6 $_{301} / \mathrm{V} 5$ and AcVP6 $311 /$ V5 were propagated as previously described [30]. All recombinant baculoviruses were propagated in Spodoptera frugiperda Sf9 cells (ATCC) grown at $27^{\circ} \mathrm{C}$ in TNM-FH medium (SIGMA) supplemented with $10 \%$ foetal bovine serum (FBS) and antibiotic-antimycotic solution (GIBCO).

\section{Expression analysis of VP6 mutants}

Sf9 cells grown in $25 \mathrm{~cm}^{2}$ flasks were infected at a multiplicity of infection (moi) of 5 with each recombinant baculovirus and 5 days post infection (dpi), cells were lysed by boiling in presence of $500 \mu \mathrm{l}$ cracking buffer $(50 \mathrm{mM}$ Tris-HCl pH 6.8, 2\% SDS, 0.01\% bromo-phenol blue, 1\% 2-mercaptoethanol, 10\% glycerol) and proteins were resolved by SDS-PAGE. Recombinant proteins were detected by Coomassie blue staining or Western blot with a commercial monoclonal antibody $(\mathrm{MAb})$ directed to the
V5 epitope (Invitrogen) or a rabbit polyclonal serum against rotavirus SA11.

\section{Analysis of the formation of VP6 trimer, multimers and VLPs}

For testing the ability of VP6 mutants to form trimers, 5 dpi infected cells grown in $25 \mathrm{~cm}^{2}$ flasks were disrupted in $500 \mu \mathrm{l}$ cracking buffer (without 2-mercaptoethanol or DTT), incubated at $37^{\circ} \mathrm{C}$ for $30 \mathrm{~min}$ and subjected to SDS PAGE under no reducing conditions [36]. Furthermore, 5 dpi infected cells were harvested in TNMC buffer, subjected to three freeze-thaw cycles, sonicated and then electrophoresed under native conditions [36]. Trimers were detected by Western blot with a MAb to V5 epitope or a rabbit polyclonal serum against rotavirus SA11.

In order to evaluate the ability of VP6 mutants to multimerize alone or interact with VP2 to form VLPs, Sf9 cells grown in four $175 \mathrm{~cm} 2$ flasks were infected or co-infected at a moi of 5 for each version of AcVP 6 and a moi of 8 for AcVP2 and 5 dpi supernatants were collected, clarified 10 min at 1,600 $\times \mathrm{g}$ and VLPs were pelleted by ultracentrifugation through a $30 \%(\mathrm{w} / \mathrm{w})$ sucrose cushion at 70,000 $\times$ $\mathrm{g}$ for $90 \mathrm{~min}$ at $10^{\circ} \mathrm{C}$. Pellets were resuspended in $300 \mu \mathrm{l}$ of TNMC buffer (10 mM Tris-HCl pH 7, $150 \mathrm{mM} \mathrm{NaCl}, 1$ $\mathrm{mM} \mathrm{MgCl}{ }_{2}, 10 \mathrm{mM} \mathrm{CaCl} 2$ ). Ten $\mu$ l were disrupted with cracking buffer and analyzed by SDS-PAGE and Western blot. Recombinant proteins were detected by a rabbit polyclonal serum against rotavirus SA11 or a mix of this serum and the Mab directed to V5. The remaining material was loaded onto a CsCl gradient (density $-1.32 \mathrm{~g} \mathrm{ml}^{-}$ 1 ) and centrifuged $134,000 \times \mathrm{g}$ for $18 \mathrm{~h}$ at $10^{\circ} \mathrm{C}$. The fraction corresponding to VLPs was collected, dialyzed against TMNC buffer and concentrated by ultracentrifugation at $70,000 \times \mathrm{g}$ for $90 \mathrm{~min}$ at $10^{\circ} \mathrm{C}$. Pellets were resuspended in $50 \mu \mathrm{l}$ of TNMC buffer and stored at $4^{\circ} \mathrm{C}$ for ELISA and electron microscopy observation.

\section{Electron microscopy}

VLPs obtained by ultracentrifugation in $\mathrm{CsCl}$ gradients were loaded onto cupper grids of a 200 mesh, stained with $2 \%$ uranyl acetate and analyzed by electronic microscopy (JEOL SVC-model) at $80 \mathrm{kV}$.

In order to determinate the heterologous peptide exposition onto recombinant VLPs surface, an immunogold labelling was performed. All incubations were carried out on drops of reagent on strips of parafilm in a covered chamber. Briefly, recombinant VLPs obtained by ultracentrifugation in $\mathrm{CsCl}$ gradients were loaded onto nickel grids and a blocking step was carried out for $30 \mathrm{~min}$ with PBS 1\% BSA. Once removed the excess buffer, the grids were incubated with a drop of monoclonal anti-V5 diluted 1/50 in blocking buffer for $1 \mathrm{~h}$ a room temperature. After each step, grids were washed five times with 
ultrapure water. Then, the samples were incubated with a drop of anti-mouse antibody conjugated to $10 \mathrm{~nm}$ gold particles (SIGMA) diluted 1/50 in blocking buffer. Finally, grids were stained in $2 \%$ uranyl acetate and analyzed by electron microscopy.

\section{Characterization of recombinant VLPs by ELISA}

Microtitration plates (Maxisorp, Nunc, Rochester, NY) were coated with $50 \mu \mathrm{l}$ of an optimal dilution $(1 / 2000)$ of a rabbit polyclonal serum against rotavirus-SA11 in carbonate buffer pH $9.6\left(\mathrm{Na}_{2} \mathrm{CO}_{3} 15 \mathrm{mM}, \mathrm{NaHCO}_{3} 35 \mathrm{mM}\right.$, $\mathrm{pH} 9,6)$, by an overnight incubation at $4{ }^{\circ} \mathrm{C}$. The next day, a blocking step was performed for $1 \mathrm{~h}$ at $37^{\circ} \mathrm{C}$ with PBS, $0.05 \%$ Tween-20, 5\% horse serum (PBS-T-HS) and 5\% non-fat milk. After each step, plates were washed five times with washing buffer (PBS, 0.05\% Tween-20). Next, a $50 \mu \mathrm{l}$ volume of a dilution of purified VP6/VP2 or recombinant $\mathrm{VP}_{171} \mathrm{~V} 5 / \mathrm{VP} 2$ VLPs $(25 \mathrm{ng} /$ well) was prepared, added it to the plate and incubated for $1 \mathrm{~h}$ at $37^{\circ} \mathrm{C}$. After this incubation, optimal sera dilutions of $\mathrm{MAb}$ against V5 epitope or mouse polyclonal against rotavirus SA11 were transferred to the ELISA plate and incubated for $1 \mathrm{~h}$ at $37^{\circ} \mathrm{C}$. Finally, $50 \mu \mathrm{l}$ of a 1:2000 dilution of horseradish peroxidase conjugated antibody (KPL, Guildford UK GU2 5GN) in PBS-T-HS were applied for $1 \mathrm{~h}$ at $37^{\circ} \mathrm{C}$. Then, $50 \mu \mathrm{l}$ of the substrate solution (ABTS $0.5 \mathrm{mg} /$ $\mathrm{ml}$ (Sigma-Aldrich, St. Louis, MO) in $0.1 \mathrm{M}$ citrate buffer, pH 4.2 containing $0.03 \%$ hydrogen peroxide) were added, and after incubation at room temperature for 20 minutes absorbance readings at $405 \mathrm{~nm}$ were determined. Each sample was tested in duplicates.

\section{Immunization and evaluation of the humoral immune response}

Female BALB/c mice (6-8 weeks old) were used for vaccination. Animals received two doses of an oil-based vaccine consisting of $300 \mathrm{ng}$ of $\mathrm{VLPsVP}_{171} \mathrm{~V} 5$ or $5 \mu \mathrm{g}$ of monomeric $\mathrm{VP}_{171} \mathrm{~V} 5$, formulated with incomplete Freund adjuvant (IFA) at days 1 and 21 by the intra-peritoneal (ip) route. A control group was immunized with VP6wt. Animals were bled at different times post-vaccination and were maintained all the time with free access to sterile food and water.

The humoral immune response against V5 epitope was evaluated by ELISA. Briefly, a recombinant not related protein CAT-V5, was produced in E. coli (using pRSET expression vector) and purified by Ni-NTA agarose (QIAGEN). Microtitration plates (Maxisorp, Nunc) were coated with $75 \mathrm{ng} /$ well of purified CAT-V5 in carbonate buffer $\mathrm{pH} 9.6$, by an overnight incubation at $4^{\circ} \mathrm{C}$. The next day, plates were blocked with PBS-T-HS and 5\% nonfat milk and subsequently incubated for $1 \mathrm{~h}$ at $37^{\circ} \mathrm{C}$ with mice serum sample (serial dilutions from 1:50 to 1:5000) and a secondary anti-mouse-IgG horseradish peroxidase conjugated antibody (KPL). Primary and secondary antibodies were diluted with PBS-T-HS. After each step, plates were washed five times with washing buffer. Finally, the reaction was developed by addition of ABTS $0.5 \mathrm{mg} / \mathrm{ml}$ (Sigma-Aldrich, St. Louis, MO) in $0.1 \mathrm{M}$ citrate buffer $\mathrm{pH}$ 4.2 containing $0.03 \%$ hydrogen peroxide, and absorbance readings at $405 \mathrm{~nm}$ were determined after incubation at room temperature. Each sample was tested in duplicate.

Titers were expressed as $\log _{10}$ of the reciprocal of the highest serum dilution, which give OD readings al least twofold higher than those of control animals.

\section{Abbreviations}

VLPs: virus-like particles; PBS-T-HS: PBS, 0.05\% Tween20, 5\% horse serum; CAT-V5: Chloranphenicol Acetyl Transferase protein fused to V5 epitope.; MAb: monoclonal antibody; OD: optical density

\section{Competing interests}

The authors declare that they have no competing interests.

\section{Authors' contributions}

AP: constructed all recombinant baculoviruses, characterized the different chimeric VP6-V5, developed a sandwich ELISA to characterize recombinant VLPs, immunized mice and evaluated the immune response by ELISA (expressed and purified a not related recombinant protein CAT-V5 in order to evaluate the antibody title against V5 epitope). PM: established optimal conditions to obtain VLPs, prepared all samples to electron microscopy (negativ stain and immunogold labelling). OT: have been involved in drafting the manuscript and have given final approval of the version to be published.

\section{Author's information}

A.P.: PhD. in Biology, University of Buenos Aires, Argentina. Assistant Research of Argentine Commission for Scientific and Technological Research (CONICET).

P.M.: Doctoral fellow of CONICET.

O.T.: PhD. in Biology, University of Buenos Aires, Argentina. Research of CONICET and Research of National Institute of Agricultural Technology (INTA).

\section{Acknowledgements}

We are grateful to Dr. Mariana Del Vas for a critical reading of the manuscript and to Julián Diodati for technical assistance with the electron microscopy. This work was supported in part by a grant from National Institute of Agricultural Technology (AEGR 24I3).

\section{References}

I. Carter J, Hagensee M, Taflin MC, Lee SK, Koutsky LA, Galloway DA: HPV-I capsids expressed in vitro detect human serum antibodies associated with foot warts. Virology 1993, 195:456-462. 
2. Kirnbauer R, Hubbert NL, Wheeler CM, Becker TM, Lowy DR, Schiller JT: A virus-like particle enzyme-linked immunosorbent assay detects serum antibodies in a majority of women infected with human papillomavirus type 16. J Natl Cancer Inst 1994, 86:494-499.

3. Roberson JA, Crill WD, Chang G]: Differentiation of West Nile and St. Louis encephalitis virus infections by use of noninfectious virus-like particles with reduced cross-reactivity. J Clin Microbiol 2007, 45:3।67-3I74.

4. Yoshii K, Ikawa A, Chiba Y, Omori Y, Maeda J, Murata R, Kariwa H, Takashima I: Establishment of a neutralization test involving reporter gene-expressing virus-like particles of tick-borne encephalitis virus. J Virol Methods 2009, 161:173-176.

5. Fothergill T, McMillan NA: Papillomavirus virus-like particles activate the PI3-kinase pathway via alpha-6 beta-4 integrin upon binding. Virology 2006, 352:319-328.

6. Sivakumar S, Wang Z, Harrison RL, Liu S, Miller WA, Bonning BC: Baculovirus-expressed virus-like particles of Pea enation mosaic virus vary in size and encapsidate baculovirus mRNAs. Virus Res 2009, 139:54-63.

7. Christensen ND, Reed CA, Cladel NM, Han R, Kreider JW: Immunization with viruslike particles induces long-term protection of rabbits against challenge with cottontail rabbit papillomavirus. J Virol 1996, 70:960-965.

8. Emeny RT, Wheeler CM, Jansen KU, Hunt WC, Fu TM, Smith JF, MacMullen S, Esser MT, Paliard X: Priming of human papillomavirus type II-specific humoral and cellular immune responses in college-aged women with a virus-like particle vaccine. J Virol 2002, 76:7832-7842

9. Garcea RL, Gissmann L: Virus-like particles as vaccines and vessels for the delivery of small molecules. Curr Opin Biotechnol 2004, I5:513-5I7.

10. Hantz S, Alain S, Denis F: [Human papillomavirus prophylactic vaccines: stakes and perspectives]. Gynecol Obstet Fertil 2006 , 34:647-655.

11. Kang SM, Yoo DG, Lipatov AS, Song JM, Davis CT, Quan FS, Chen LM, Donis RO, Compans RW: Induction of long-term protective immune responses by influenza $\mathrm{H} 5 \mathrm{NI}$ virus-like particles. PLOS ONE 2009, 4:e4667.

12. O'Neal CM, Crawford SE, Estes MK, Conner ME: Rotavirus viruslike particles administered mucosally induce protective immunity. J Virol 1997, 71:8707-87/7.

13. Suzich JA, Ghim SJ, Palmer-Hill FJ, White WI, Tamura JK, Bell JA, Newsome JA, Jenson $A B$, Schlegel R: Systemic immunization with papillomavirus LI protein completely prevents the development of viral mucosal papillomas. Proc Natl Acad Sc USA 1995, 92: II553-II557.

14. Warfield KL, Swenson DL, Olinger GG, Kalina WV, Aman MJ, Bavari $S$ : Ebola virus-like particle-based vaccine protects nonhuman primates against lethal Ebola virus challenge. J Infect Dis 2007 , 196(SuppI 2):S430-437.

15. Grgacic EV, Anderson DA: Virus-like particles: passport to immune recognition. Methods 2006, 40:60-65.

16. Fifis T, Gamvrellis A, Crimeen-Irwin B, Pietersz GA, Li J, Mottram PL, McKenzie IF, Plebanski M: Size-dependent immunogenicity: therapeutic and protective properties of nano-vaccines against tumors. J Immunol 2004, 173:3 I 48-3I 54

17. Fields B, Knipe D, Howley P: Field's Virology 3rd edition. LippincottRaven, Philadelphia; 1996.

18. Kapikian AZ: Viral gastroenteritis. Jama 1993, 269:627-630.

19. Kapikian AZ, Flores J, Hoshino Y, Glass RI, Midthun K, Gorziglia M, Chanock RM: Rotavirus: the major etiologic agent of severe infantile diarrhea may be controllable by a "Jennerian" approach to vaccination. J Infect Dis 1986, 153:8I5-822.

20. Crawford SE, Labbe M, Cohen J, Burroughs MH, Zhou Y], Estes MK: Characterization of virus-like particles produced by the expression of rotavirus capsid proteins in insect cells. J Virol 1994, 68:5945-5952.

21. Gonzalez SA, Affranchino JL: Assembly of double-layered viruslike particles in mammalian cells by coexpression of human rotavirus VP2 and VP6. J Gen Virol I995, 76(Pt 9):2357-2360.

22. Labbe M, Charpilienne A, Crawford SE, Estes MK, Cohen J: Expression of rotavirus VP2 produces empty corelike particles. Virol 1991, 65:2946-2952.
23. Zeng CQ, Labbe M, Cohen J, Prasad BV, Chen D, Ramig RF, Estes MK: Characterization of rotavirus VP2 particles. Virology 1994 , 201:55-65.

24. Lepault J, Petitpas I, Erk I, Navaza J, Bigot D, Dona M, Vachette P, Cohen J, Rey FA: Structural polymorphism of the major capsid protein of rotavirus. Embo J 200I, 20:1498-I507.

25. Banos DM, Lopez S, Arias CF, Esquivel FR: Identification of a Thelper cell epitope on the rotavirus VP6 protein. J Virol 1997 , 7I:419-426.

26. Esquivel FR, Lopez S, Guitierrez XL, Arias C: The internal rotavirus protein VP6 primes for an enhanced neutralizing antibody response. Arch Virol 2000, I45:8/3-825.

27. Frenchick PJ, Sabara MI, Ready KF, Babiuk LA: Biochemical and immunological characterization of a novel peptide carrier system using rotavirus VP6 particles. Vaccine 1992, 10:783-791.

28. Redmond MJ, Ohmann HB, Hughes HP, Sabara M, Frenchick PJ, Poku SK, ljaz MK, Parker MD, Laarveld B, Babiuk LA: Rotavirus particles function as immunological carriers for the delivery of peptides from infectious agents and endogenous proteins. Mol Immunol |99|, 28:269-278.

29. Southern JA, Young DF, Heaney F, Baumgartner WK, Randall RE: Identification of an epitope on the $P$ and $V$ proteins of simian virus 5 that distinguishes between two isolates with different biological characteristics. J Gen Virol I99|, 72(Pt 7): I55I-I557.

30. Affranchino JL, Gonzalez SA: Deletion mapping of functional domains in the rotavirus capsid protein VP6. J Gen Virol 1997. 78(Pt 8): 1949-1955.

31. Mena JA, Ramirez OT, Palomares LA: Quantification of rotaviruslike particles by gel permeation chromatography. J Chromatogr B Analyt Technol Biomed Life Sci 2005, 824:267-276.

32. Clapp LL, Patton JT: Rotavirus morphogenesis: domains in the major inner capsid protein essential for binding to singleshelled particles and for trimerization. Virology 1991, 180:697-708.

33. Mathieu M, Petitpas I, Navaza J, Lepault J, Kohli E, Pothier P, Prasad BV, Cohen J, Rey FA: Atomic structure of the major capsid protein of rotavirus: implications for the architecture of the virion. Embo J 200I, 20: I 485-I497.

34. Prasad BV, Wang GJ, Clerx JP, Chiu W: Three-dimensional structure of rotavirus. J Mol Biol I988, 199:269-275.

35. O'Reilly DR, Miller LK, Luckow VA: Baculovirus expression vectors. A laboratory manual. 1994.

36. Auseber F, Brent R, Kingston R, Moore D, Seidman J, Struhc K: Current protocols in molecular biology John Wiley and Sons, Inc edn; 1994.

Publish with Bio Med Central and every scientist can read your work free of charge

"BioMed Central will be the most significant development for disseminating the results of biomedical research in our lifetime. "

Sir Paul Nurse, Cancer Research UK

Your research papers will be:

- available free of charge to the entire biomedical community

- peer reviewed and published immediately upon acceptance

- cited in PubMed and archived on PubMed Central

- yours - you keep the copyright 